\title{
An international forum for discussion of gastric cancer is needed, particularly now
}

\author{
TAKeshi Sano \\ Gastric Surgery Division, National Cancer Center Hospital, 5-1-1 Tsukiji, Tokyo 104-0045, Japan
}

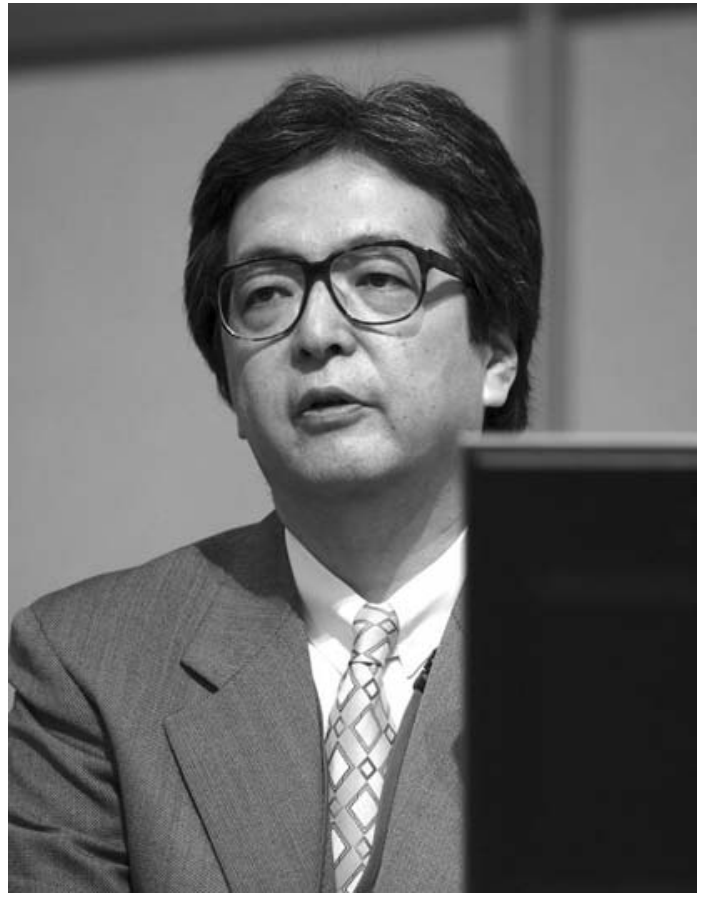

Takeshi Sano, M.D.

Head, Gastric Surgery Division

National Cancer Center Hospital, Tokyo

Gastric cancer may or may not be changing. According to the estimate by the International Agency for Research on Cancer, the worldwide incidence of gastric cancer in 2002 was 933,937 , of which no less than $56 \%$ of cases were from East Asia (China, Japan, and Korea) alone. Interestingly, gastric cancer in this high-incidence area is not, or only very slowly, undergoing the phenomenon of "proximal shift" of tumors, which today is a definite trend in gastric cancer in the West. The difference between East and West in terms of tumor biology as well as treatment modality seems to be becoming greater over the years. Now many physicians and researchers in both hemispheres are wondering if they are fighting against the same disease that the other side is.

In such a situation, the role of the International Gastric Cancer Association (IGCA) is particularly important. It provides opportunities for worldwide specialists to meet and discuss the disease. Our journal, Gastric Cancer, should also be a forum for global discussion as the only international journal specialized in this malignancy.

Since its first publication in 1998, Gastric Cancer has maintained some unique features as the joint official journal of IGCA and the Japanese Gastric Cancer Association (JGCA). The editorial board consists of the same number of members from both associations, and any submitted manuscript is reviewed by a team composed of referees from both associations. Even the position of Editor-in-Chief has been shared; Professor J. Rüdiger Siewert from IGCA and Dr. Oichiro Kobori from JGCA formed a good partnership to develop a high-quality peer-review system.

Now I have succeeded Dr. Kobori to undertake the JGCA's part as an Editor-in-Chief. My task is clear: to further expand the journal's scope as a forum for global discussion and to enhance the journal's status in the field of oncology.

Both Professor Siewert and I are surgeons. Approximately $80 \%$ of the JGCA and IGCA members are also surgeons. However, our journal is not a surgical journal. Our editorial board has prominent medical oncologists and scientists to welcome any category of papers related to gastric cancer. Our goal is comprehensive understanding and consequent conquest of gastric cancer.

In 2004, a total of 1,450 English papers having the keyword "stomach neoplasms" were published, according to the PubMed database. Of these, 513 papers $(35.4 \%)$ were from Japanese institutions or had at least one Japanese author. Considering the high incidence of 
the disease in Japan, this high proportion is understandable. Gastric Cancer is a quarterly journal, and we published 41 papers in 2004. This accounted only for $2.8 \%$ of the above-mentioned 1,450 , and I would aim at doubling this within 3 years. Of all 268 papers published in volumes 1 to 7 of Gastric Cancer, 207 (77.2\%) were from Japanese institutions. Although this high proportion is due largely to the fact that the membership ratio of JGCA to IGCA is 6 to 1 , I hope we will receive more papers from outside Japan to make our journal a truly international forum.

As a gastric surgeon, I have visited 22 countries either for giving lectures or live demonstrations of surgery. As a host at the National Cancer Center Hospital, Tokyo, I have received visitors from an additional 21 countries and had intensive discussions with all of them. I believe I am part of a global network of gastric cancer specialists, on a personal level, and I intend to make full use of that network to increase the value of the journal.
Dear colleagues: Please do not hesitate. You have good reasons to write papers for Gastric Cancer:

1. Your paper will be sent directly to 6,000 worldwide specialists of gastric cancer.

2. The abstract of your paper is immediately listed in Medline and PubMed.

3. The PDF file of your paper is uploaded on the Springer website and can be downloaded through their 4,500 worldwide access sites in addition to downloading by our 6,000 subscribers.

4. Your paper may be selected for the Nishi Award of the JGCA (the best three papers each year) and you will be awarded US $\$ 2,000$.

I am looking forward to your participation in this international forum for gastric cancer.

Takeshi Sano Editor-in-Chief 